\title{
Role of Computed Tomography Scan in Non-Localized Headache in Tertiary Hospital of Mid Western Region of Nepal
}

\author{
Ghimire $\mathrm{P}^{1}$, Singh $\mathrm{BP}^{2}$, Chaturvedi SK ${ }^{3}$
}

\begin{abstract}
Objective: To investigate the implication of a computed tomography scan for headache with non-localizing sign. Materials and methods: One hundred and thirty six patients with headache having non-localizing signs were included in this prospective study. Patients with age $>11$ years, incomplete radiological or clinical data, recent/new onset headache, any immunosuppressive state, neurological deficits at the time of presentation, history of fever, trauma, any previous surgical intervention and any malignancy were excluded from the study. Results: Among the 136 patients, $73 \%$ were females and $27 \%$ were males with age range of $11-76$ years. Negative computed tomography scan was present in $91(66.9 \%)$ cases. Positive findings that significantly influenced the management were present in only $6(4.4 \%)$ cases. Conclusion: Computed tomography in headache with non-localizing signs has a poor yield for a significant intracranial pathology. A careful and detailed assessment curtails the need for inadvertent imaging thus reducing the economic burden and health related hazards.
\end{abstract}

Key words: Computed tomography, headache, radiation, non-localizing, imaging

\section{INTRODUCTION}

Headache is a common clinical presentation in patients presenting in the emergency department causing significant morbidity ${ }^{1}$. Imaging has been widely performed for evaluation of headache and various studies have demonstrated a very lowyield of significant pathologies. A detailed history and physical examination can avoid unnecessary imaging in patients presenting with no ominous symptoms and non-localizing neurological signs ${ }^{2}$. Inadvertent radiological imaging causes significant economic burden as well as exposure to radiations ${ }^{3}$. In this study, we attempted to correlate the implication of computed tomography scan in patients with headache without localizing signs in resource-strained settings.

\section{MATERIAL AND METHODS}

This is a prospective longitudinal study conducted in the department of radiology and imaging at Nepalgunj Medical College and Teaching Hospital, Kohalpur, Banke, Nepal for all computed tomography scans performed for non localized headaches during period between December 2011 to October 2012. The inclusion criteria for the study included i) No neurological deficits at the time of presentation ii) no history of fever, trauma, any previous surgical intervention iii) no history

1. Dr. Prasanna Ghimire

2. Prof. B. P. Singh

3. Prof. S. K. Chaturvedi

\section{Address for correspondence:}

Dr. Prasanna Ghimire

Department of Radiology

Nepalgunj Medical College Teaching Hospital

Kohalpur, Banke, Nepal

Email: drprasannaghimire@gmail.com of any malignancy. The exclusion criteria included (i) age of patient $>11$ years (ii) incomplete radiological or clinical data (iii) recent/new onset headache (iv) any immunosuppressive state.

Amongst 164 patients, only 136 patients were included in the study that fulfilled both the inclusion and exclusion criteria. A standard head CT protocol ( $120 \mathrm{k} \mathrm{V}, 380 \mathrm{mAs}$ ) with sequential scans of $5 \mathrm{~mm}$ from the level below the foramen magnum through the vertex of the skull was performed. CT scans were reviewed by two radiologists in consortium and a final opinion was made. The results were divided into three groups adopted as in the study by Jordan et al into (1) no intracranial abnormality (negative study); (2) clinically significant intracranial abnormality, such as space-occupying lesions or intracranial hemorrhage and (3) positive without clinical significance or with extracranial abnormality. The patients were followed up for a period of 6 months from the date of study. Statistical analyses were performed using SPSS 16.0.

\section{RESULTS}

The age of the patients ranged from 11 years to 78 years with mean age of presentation for male (44.24 yrs of age) being higher than for female (42.12 years) though not statistically significant. There were 98 females and 38 males with female to male ratio were 2.67: 1 . There were negative $\mathrm{CT}$ findings in 91 scans accounting to $66.9 \%$ of the total study and thus classified as Group 1. Among the positive findings on CT, Group 2 which included clinically significant findings that influenced management intensively was only $6(4.4 \%)$ cases with a female preponderance in the age group of 31-40 years. There were 2 brain tumors (1 low grade glioma, 1 cerebral metastasis), 1 subdural hemorrhage, 1 sub-arachnoid hemorrhage with hydrocephalus and 2 arterio-venous malformations. There were $39(28.7 \%)$ cases which had positive findings that did not 
influence the management. These included cerebral atrophy (12 cases), acute and chronic rhinosinusitis (10 cases), chronic infarct/encephalomalacia (8 cases), calcified granulomas (9 cases).

\section{DISCUSSION}

Headache is one of the commonest symptoms for patient presentation in the emergency department worldwide ${ }^{1}$. Although, majority of the causes of headache are benign in etiology, there has been an exponentially increased number of routine neuroimaging studies in recent times ${ }^{2-4}$. The age of presentation in our study was at advanced age of male compared to female counterparts. This however seems to be due to increased number of female cases in our study which is similar to previous studies.

Our present study demonstrates a very low yield of positive cases in radiological studies performed for headaches with non-localizing signs. The positive CT scan studies that significantly influenced the management of the patient in our study that required immediate surgical intervention was low; only $6(4.4 \%)$ cases amongst 136 cases which is complying to a previous study done by Al-Nabhani, K., et al ${ }^{5}$.

The positive cases that did not influence the management and were unrelated to the headache primarily included cerebral atrophy, sinusitis, chronic infarcts/ encephalomalacia, calcified granulomas, intracranial lipomas which are in keeping with previous studies demonstrating similar findings ${ }^{6}$. There are many limitations to our study. Firstly, our study was in a limited number of cases. Besides, we have not stratified the cases depending on the referring specialty.

Studies have shown that detailed clinical history and physical examination significantly increased the yield in radiological

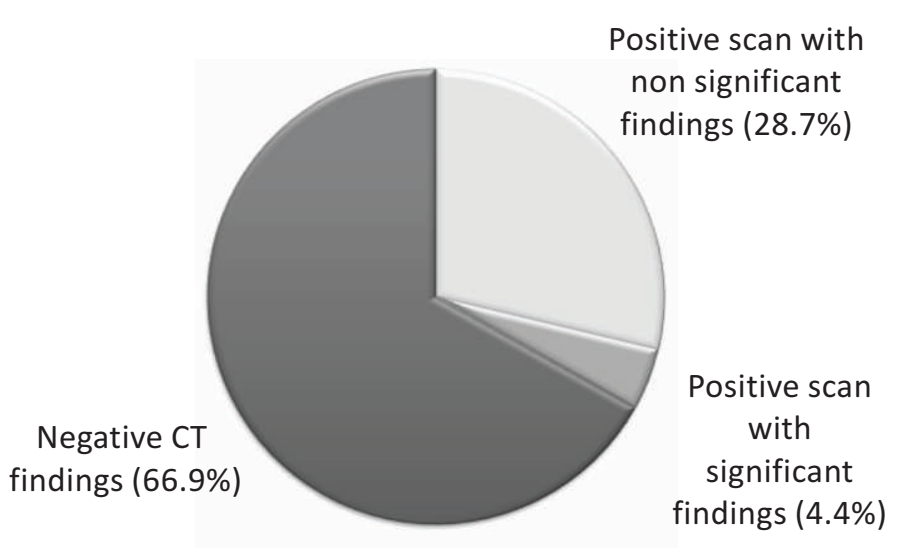

Figure 1: CT findings in headache

studies with highest among specialist neurologist ${ }^{6}$. The consequences of "overuse" neuroimaging has a substantial cost causing economic burden to health seeking individuals especially in resource constrained setting in developing countries. Various guidelines have been recommended against routine imaging in patients with headaches. There are multiple factors that however influence the implementation of these guidelines.

Patient's apprehension and anxiety, medico-legal reasons and physicians over concern of missing an intracranial pathology are some of the element for this inadvertent neuroimaging utilization ${ }^{8}$. Certain studies have shown that educating patients regarding unwarranted testing and the potential radiation hazards of the so- low yield test can be useful to curb utilization and optimize neuroimaging practices.

\begin{tabular}{|c|c|c|c|c|c|c|}
\hline Age Group & \multicolumn{2}{|c|}{ Group 1 } & \multicolumn{2}{c|}{ Group 2 } & \multicolumn{2}{c|}{ Group 3 } \\
\hline (in years) & Male & Female & Male & Female & Male & Female \\
\hline $11-20$ & 2 & 3 & 0 & 0 & 1 & 2 \\
\hline $21-30$ & 3 & 15 & 0 & 1 & 2 & 4 \\
\hline $31-40$ & 6 & 17 & 1 & 1 & 2 & 6 \\
\hline $41-50$ & 4 & 11 & 0 & 2 & 2 & 7 \\
\hline $51-60$ & 4 & 7 & 0 & 1 & 2 & 3 \\
\hline $61-70$ & 3 & 8 & 0 & 0 & 2 & 2 \\
\hline $71-80$ & 2 & 6 & 0 & 0 & 2 & 2 \\
\hline TOTAL & $\mathbf{2 4}$ & $\mathbf{6 7}$ & $\mathbf{1}$ & $\mathbf{5}$ & $\mathbf{1 3}$ & $\mathbf{2 6}$ \\
\hline
\end{tabular}

Table 1. Categorization of patients based on CT scan findings.

Note: Group (1) No intracranial abnormality (negative study) Group (2) Clinically significant intracranial abnormality, such as space-occupying lesions or intracranial hemorrhage and Group (3) Positive without clinically significant intracranial pathology or with extracranial abnormality. 


\begin{tabular}{|l|l|}
\hline Group II & Brain tumors (one low-grade glioma and one metastases) [2 cases] \\
(6 cases) & AV malformations [2 cases] \\
& Subdural hemorrhage [1 case] \\
& Subarachnoid hemorrhage [1 case] \\
\hline \multirow{3}{*}{ Group III } & Cerebral atrophy [12 cases] \\
(39 cases) & Acute and chronic rhinosinusitis [10 cases] \\
& Chronic infarct/ encephalomalacia [8 cases] \\
& Calcified granulomas [9 cases] \\
\hline
\end{tabular}

Table II: CT findings in positive cases

\section{CONCLUSION}

Cranial CT examination in patients for headache with nonlocalizing signs has a very low yield for a significant intracranial pathology. A guideline should be devised based on the local resources in a developing country which would lead to high yield of positive cases without substantially increasing health care expenditure.

\section{REFERENCES}

1. Stovner L, Hagen K, Jensen R, Katsarava Z, Lipton R, Scher A, et al. The global burden of headache: a documentation of headache prevalence and disability worldwide. Cephalalgia : an international journal of headache. 2007;27(3):193-210.

2. Evans RW. Diagnostic testing for migraine and other primary headaches. Neurologic clinics. 2009;27(2):393-415.

3. Fazel R, Krumholz HM, Wang Y, Ross JS, Chen J, Ting HH, et al. Exposure to low-dose ionizing radiation from medical imaging procedures. The New England journal of medicine. 2009;361(9):849-57.

4. Callaghan BC, Kerber KA, Pace RJ, Skolarus LE, Burke JF. Headaches and neuroimaging: High utilization and costs despite guidelines. JAMA Internal Medicine. 2014;174(5):819-21.

5. Al-Nabhani K, Kakaria A, Syed R. Computed tomography in management of patients with non-localizing headache. Oman medical journal. 2014;29(1):28-31.

6. Imarhiagbe FA, Ogbeide E. Should non acute and recurrent headaches have neuroimaging before review by a Neurologist?-a review in a Southern Nigerian Tertiary Hospital. Annals of African medicine. 2011;10(4):290-3.

7. Jordan JE, Ramirez GF, Bradley WG, Chen DY, Lightfoote JB, Song A. Economic and outcomes assessment of magnetic resonance imaging in the evaluation of headache. Journal of the National Medical Association. 2000;92(12):573-8.

8. Sun $\mathrm{Z}, \mathrm{Ng} \mathrm{KH}$, Vijayananthan $\mathrm{A}$. Is utilisation of computed tomography justified in clinical practice? Part I: application in the emergency department. Singapore Med J. 2010;51(3):200-6. 